\title{
Effects of External Conceptual Models and Verbal Explanations on Shared Understanding in Small Groups
}

\author{
Wolfgang Maass ${ }^{1,3}$, Veda C. Storey ${ }^{2}$, and Tobias Kowatsch ${ }^{3}$ \\ ${ }^{1}$ Saarland University, 66123 Saarbrücken, Germany \\ ${ }^{2}$ University Plaza, Georgia State University, Atlanta 30399 United States \\ ${ }^{3}$ Institute of Technology Management (ITEM), University of St. Gallen, Switzerland \\ wolfgang.maass@iss.uni-saarland.de, vstorey@gsu.edu, \\ tobias.kowatsch@unisg.ch
}

\begin{abstract}
Effective conceptual modeling requires a shared understanding of the concepts that are found in an application domain. Achieving such understanding, especially for large design problems, is a challenging, and long-standing problem. Conceptual models tend to be either subjective representations of individuals that require mutual knowledge sharing between members of a modeling team or externalized normative representations that require knowledge transfer from model preceptors to model receptors. Model preceptors have either created a conceptual model or conceived it by another preceptor. In prior studies, normative conceptual models were used to investigate knowledge transfer between preceptors and receptors. This research, in contrast, investigates knowledge transfer of conceptual models between model owners and receptors. A $2 \times 2$ study design with modeling novices was used that varied the type of conceptual modeling language and the type of information system. Further testing investigated whether knowledge transfers were affected by additional verbal explanations given by the preceptor. Each modeler was provided access to two conceptual modeling languages that naturally support structure or process representations. The study investigated whether the use of particular conceptual modeling languages differ in their effects on shared understanding between two persons and whether additional verbal explanations might increase shared understanding. The results of this exploratory empirical study provide useful insights into the use of Conceptual Modeling Language pairs for shared understanding in conceptual modeling in small groups.
\end{abstract}

\section{Introduction}

The conceptual modeling phase of systems development involves the process of abstracting the real world to represent it by a model that focuses on key entities and relationships of an application domain [1]. Conceptual models that accurately represent an application domain provide a critical means for shared understanding in an information system development team [2, 3]. A long-standing challenge in information systems research is that many information systems projects fail either completely or partially [2, 4]. Prior studies have concluded that mis-understanding between team members is one of the key issues for such failures [5, 6]. People from 
different departments use incompatible languages for expressing their ideas and understandings of the envisioned information system [7]. Software engineers, for example, use UML tools, database modelers use ER diagrams; graphical designers are accustomed to scribbles and storyboards; and people from marketing use textual descriptions on customer relationship measurements.

There have been few empirical studies on understanding these communication problems in terms of the social processes associated with conceptual modeling [2]. Little empirical research has been conducted that examines how different conceptual modeling languages (CML) support shared understandings within modeling teams. Instead, researchers have focused on how normative conceptual models represented by a particular CML support individual understanding of a conceptual model (CM) [3]. In such studies, CMs were created and evaluated by modeling experts. In practice, however, CMs are rarely created by modeling experts; rather, they are created quite loosely for transferring individual understandings of a subjective CM to another team member. It is an open research question as to how non-experts use various CML for creating CMs and then use these CMs for creating a shared understanding with other non-experts.

The objective of our exploratory research is to examine the subjective side of understanding the conceptual modeling process with emphasis on supporting shared understanding amongst information systems non-expert modelers. To do so, an empirical analysis is carried out on small groups participating in a design task. The contribution of the research is to understand whether (and how) different types of CML with additional verbal explanations can assist small groups of non-experts in a shared understanding of domain concepts in order to create effective conceptual models.

\section{Theoretical Background}

\subsection{Conceptual Models}

A conceptual model expresses the meaning of terms and concepts used by domain experts to discuss a design problem, and to identify the relationships among different concepts [8]. The conceptual modeling phase of information systems development is intended to clarify the meaning of various terms, and to ensure that problems with different interpretations do not occur. The conceptual model is mapped into the physical design for implementation. It is, thus, important that conceptual models are well defined and represent the essence of an application domain. A conceptual model can be described using various notations, such as UML or OMT for object modeling, or IE or IDEF1X for entity-relationship models [9].

Regardless of the notation, it is crucial that the essence of the domain be accurately captured. The process involved in conceptual modeling involves collaboration between requirements engineering (RE) participants (e.g., user-representatives and systems analysts) where knowledge regarding the system requirements is shared, absorbed, and constructed. The main challenge of the collaborative interaction is for the participants to arrive at a shared frame of reference and conceptualization regarding system requirements. This requires understanding the process of "collaborative sense-making 
and knowledge transfer that results in the convergence of diverse mental models" [2]. Conceptual models are complex knowledge structures that can be normative versus subjective; semi-formal versus formal, external (documented) versus internal (mental) representations; and individual understanding and mental models versus cross understanding and shared understanding.

Several CML have been proposed that focus on (1) business process modeling (e.g., [10]), (2) general software engineering (e.g., [11]), (3) semantic data models (e.g., [12]), and meta-data models and computational ontologies [13]. Grammars provided by CMLs require ontologies for defining the fundamental entities and structures that should be focused by CMs [14].

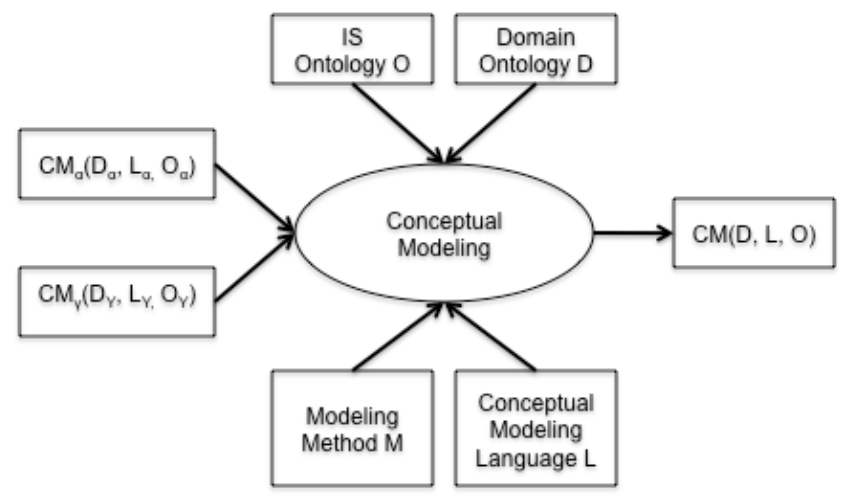

Fig. 1. Generic model of conceptual modeling

Figure 1 provides a generic model for conceptual modeling. In an idealized form, conceptual modeling transforms existing external or internal CMs into an integrated $\mathrm{CM}(\mathrm{D}, \mathrm{L}, \mathrm{O})$, by means of a modeling method $\mathrm{M}$ and a conceptual language $\mathrm{L}$ based on a domain ontology $\mathrm{D}$ and a fundamental information systems ontology $\mathrm{O}$.

Initially a CM is a subjective conceptualization of a domain created by an individual, also called a mental model [15]. By explication processes and a CM language (CML), an individual translates his/her subjective CM into an external CM. CML support informal, semi-formal, or formal representations. External conceptual models are either used in knowledge transfers or knowledge sharing activities. Knowledge transfer situations are governed by principal-agent settings between preceptors and receptors and thus use external CM in a normative manner. Normative CMs are either explained to receptors by the creator of this external CM or by trained preceptors. In the latter case, the external CM supports indirect knowledge transfers. If knowledge transfers are based on a normative CM alone, it will support individual understanding of an objective conceptualization whereas a receptor's understanding of the creator's model must be derived by additional reasoning. Indirect knowledge transfers of normative conceptual models without additional explanations by preceptors has been the core focus of empirical studies (e.g., [16]).

It could be argued that additional explanations given by a preceptor might provide additional cues that allow receptors to directly gain an understanding of the creator's 
subjective CM. Davidson argues that the theoretic assumption of knowledge transfers based on normative CMs contradict the reality of requirements elicitation, often described as "chaotic and non-linear" [17] and non-deterministic [2]. This means that external CM are not isolated but embedded into a communication discourse between various members of a group. Because conceptual models based on current CML are static descriptions, it is reasonable to expect that, at least for some domains and organizational settings, knowledge transfers can be improved by additional verbal explanations and discussions.

\subsection{Design and Mental Models}

Based on a representational theory of the mind [18], the concept of an individual mental model has been introduced that represents models of real-world or idealistic situations. Mental models are representations that are formed by individuals as internal descriptions of perceived and imagined situations [19]. For representations of perceived situations, mental models are conceived as model representations of external realities that can be used for mental processing such as mental rotations [20, 21]. During the design phases of an Information System, an internal CM is a mental model that cannot be derived by direct perception but from auxiliary descriptions such as narratives and diagrams. For framing the general problem during early design stages, narratives are more helpful representations for qualitative and ambiguous descriptions than graphics [22]. However, diagrammatic representations are very helpful during later design phases. Therefore, we assume that external CMs in the form of narratives are used early for conceiving a basic internal CM that can be enhanced by subsequently given diagrammatic CM.

\subsection{Shared Understanding}

Shared understanding should help to produce a better CM, by helping the people involved in the design realize a shared mental model, i.e. similar individual mental models [23]. This is especially important in designs that require a group effort in the requirements analysis and conceptual modeling phases. Group performance, in general, depends on interactions between group members for choosing goals and objectives, selecting solutions to achieve the group's goals, resolving conflicts, and performing activities that help to achieve group tasks [24]. These interactions require communication between group members that are intended to promote group behavior [25]. In the requirements elicitation phase, textual and diagrammatic CML have been created that facilitate communication and subsequent shared understanding by building shared mental models between members of a design team [2, 3].

Effective group performance requires that groups hold common or overlapping mental representations (referred to as shared mental models or team mental models), of goals, task requirements, procedures, role responsibilities, and situations [23]. Shared experience and information is the basis for further information sharing and, thus, strengthens understanding and collaboration within a team [26]. With respect to requirements engineering, shared mental models are mental representations of a conceptual model that are assumed by group members to be mutually agreed upon and used as a basis for group behavior, such as consensus building, problem solving, decision making, and inferencing [27, 28]. 


\section{Research Model and Hypotheses}

In the following, we describe an exploratory study that investigates the effects of different conceptual modeling languages (CMLs) on individual and shared understanding in small information systems (IS) design groups. By adopting a nonnormative stance, we also investigate the role of CML for knowledge transfer between team members.

The key questions addressed are:

- How do different CML affect individual understanding and shared understanding?

- How do textual explanations of external CM affect individual and shared understanding?

- Is shared understanding on CM affected by different types of information systems?

Our study is framed by the following constraints:

- How do external conceptual models support a shared understanding in nonnormative situations with low heterogeneity of prior modeling knowledge between members of small teams?

- How do additional verbal explanations affect this shared understanding (same moderating factors).

\section{Research Method}

This research tests 'surface understanding' [16] in the sense of shared understanding. If shared understanding is missing, problem-solving is not possible. An external conceptual model is not tested because the study is intended to test how CM and explanations affect knowledge transfers in a small team from an originating modeler to a model user. Otherwise, we would need to test how a normative, objective CM would be explained by one person to another.

\subsection{Study Design}

A laboratory experiment with two-person teams was carried out to investigate our research questions. Each subject was given one of the following two situations representing different types of IS, i.e. one Online IS and one Ubiquitous IS:

- Online IS: Michael is overweight and suffers from hazelnut allergy. Today he wants to order at his preferred online restaurant FirstMeal that supports him during his diet program. Due to his profile a series of salads and several vegetarian dishes, including such vegetarian pizza and potato dishes, are proposed. He opts for a vegetarian pizza and chooses an additional tofu topping from a list. Because he has met his last week's training program, he is awarded to choose a complimentary dessert from a menu. He opts for a smoothie with mango-coconut flavor from the category of lactose-free desserts. 
- Ubiquitous IS: Anna gets site-specific weather information when she is brushing her teeth in the bathroom. Based on weather information and her calendar, freetime event suggestions are given, e.g. "Today, 8 p.m. - Miss Marple Night at CinemaOne. Do you want to order tickets?"

With ubiquitous computing technologies [29], information services can be integrated in any kind of physical environment and provide information to users when they enter these environments. These technologies provide the basis for the class of information systems, called ubiquitous information systems [30], that support individual users and groups by providing information and communication services adapted to dynamically changing situations and user needs. Ubiquitous information systems are appropriate to include because of the increase in ubiquitous computing, emphasis on interorganizational applications, and demands for shorter project life-cycles which have introduced new techniques and changed the risk profile of requirements development project [31].

To develop an external model of these situations, two conceptual modeling languages (CML) were introduced to the subjects. The first CML combines ontologies and UML activity diagrams, both of which are denoted as CML1. On one hand, ontology describes subsumptions and other relationships between concepts, i.e. it reflects explicitly formal specifications of the terms in the domain of question and relationships among them [32]. Sharing common understanding of the structure of information among people or software agents is one of the more common goals in developing ontologies $[32,33]$. On the other hand, UML activity diagrams describe interactions between instances of concepts over time that are also called workflows (cf. OMG 2011, http://www.uml.org).

The second CML combines the notations of UML use case diagrams and sequence diagrams. This combination is denoted as CML2. UML use cases show a set of use cases and actors with an association between each interacting pair of actor and use case [34]. In contrast, a UML sequence diagram shows how messages within an IS are exchanged by considering the order of interactions (cf. OMG 2011, http://www.uml.org). Both CMLs have been selected as they provide a tool to model: (1) general structures and static relations of an IS (Ontology and UML use case diagram notations); and (2) workflows within an IS (UML activity diagrams and UML sequence diagrams).

The study was carried out in three steps. First, both subjects were asked to model one IS from one of the two situations with a given CML separately. To account for the bias that would appear from a static IS-CML combination, subjects in a team were assigned to crosswise different IS-CML combinations. Each subject was given a short introduction to the corresponding CML and exemplary diagrams were presented. By this procedure, each subject was able to become accustomed to the assigned CML. Then, each subject was allowed 30 minutes to develop an external model of the assigned situation with pen and paper. In the second step of the survey, each subject had to evaluate the teammate's model without any further explanations with regard to unambiguity, consistency and understandability of the model. These constructs were derived from [35] and corresponding questionnaire items have been created (cf. Table 2). 
In the third and final step of the survey, each subject had to explain his or her own model in no more than five minutes to his or her teammate. Then, again, each subject had to evaluate the teammate's model with regard to the items given in Table 2. In addition to demographical data, two constructs were also measured during this step: first, the extrinsic power of a CML for an individual understanding of the future IS regarding the given situation and second, the extrinsic power of a CML for a shared (team) understanding. In summary, the experimental design is shown in Table 1 and the questionnaire items are given in Table 2. All questionnaire items were rated on 7 point Likert scales ranging from strongly disagree (1) to strongly agree (7).

Table 1. Study design. Note: 34 subjects were randomly assigned to the four groups

\begin{tabular}{|c|c|c|c|}
\hline & & \multicolumn{2}{|c|}{ Type of Information System } \\
\hline & & Online IS & Ubiquitous IS \\
\hline \multirow{2}{*}{$\begin{array}{c}\text { Type of } \\
\text { Conceptual } \\
\text { Modeling } \\
\text { Language } \\
\text { (CML) }\end{array}$} & $\begin{array}{l}\text { CML1: Ontology \& UML } \\
\text { activity diagram }\end{array}$ & $\begin{array}{l}\text { Group } 1 \\
(\mathrm{n}=9)\end{array}$ & $\begin{array}{l}\text { Group } 2 \\
(\mathrm{n}=9)\end{array}$ \\
\hline & $\begin{array}{c}\text { CML2: UML use case } \\
\text { diagram \& sequence diagram }\end{array}$ & $\begin{array}{l}\text { Group } 3 \\
(n=8)\end{array}$ & $\begin{array}{l}\text { Group } 4 \\
(n=8)\end{array}$ \\
\hline
\end{tabular}

\subsection{Results and Discussion}

Ten female and 24 male students from a media informatics department participated in the lab experiment. Their age ranged between 19 and $24(n=25)$ and between 25 and $29(n=6)$. Three subjects were over 30. The subjects understood the semantics and practical application of the CMLs (Mean: 5.76; SD: .89) and judged themselves to be competent in evaluating the corresponding external conceptual models (Mean: 5.32; SD: 1.06). Overall, the instructions of the study have been understood (Mean: 5.53; SD: .89) and the length of the study was perceived acceptable (Mean: 4.26; SD: 1.56). The descriptive statistics of all constructs are presented in Table 2.

Paired-sample t-tests were used to identify any significant differences of evaluations before and after an explanation was provided regarding one particular external CM. The results indicate that explanations significantly increased comprehensibility of the external CMs for both types of information systems (cf. COM5-9 in Table 2).

Furthermore, high mean values on unambiguity and comprehensibility indicate that the subjects perceived the provided $\mathrm{CM}$ as a sufficient basis for deriving an internal $\mathrm{CM}$ of high quality. With respect to consistency, no significant change was found when test persons received additional explanations. For consistency, in general, it can be argued that structural and process elements are well captured by Use cases / sequence diagrams and ontologies / activity diagrams alike. Only for Ubiquitous IS and CML2 (UML use case diagrams \& sequence diagram) was the consistency of the external CMs judged higher after the explanation (cf. CON4/6/8 in Table 2). This could be explained by deficiencies of use case diagrams / sequence diagrams with respect to modeling domains for UIS. 
Table 2. Instrument and descriptive statistics. Note: $\mathrm{SD}=$ standard deviation, $\mathrm{MO}=$ Model Only, $\mathrm{M}+\mathrm{E}=$ Model + Explanation, Situation $1=$ Online IS, Situation $2=$ Ubiquitous IS; $* / * *$ $=.05 / .01$ significance levels for paired-sample $\mathrm{t}$-test $(\mathrm{MO}$ versus $\mathrm{M}+\mathrm{E})$.

Construct

Unambiguity: I think this model of type [CML1/2] accurately represents Situation [1/2].

\begin{tabular}{l|l|l} 
UNA1: CML1 + Online IS $(\mathrm{n}=9)$ & $6.11(0.60)$ & $5.78(1.20)$ \\
\hline UNA2: CML1 + Ubiquitous IS $(\mathrm{n}=9)$ & $6.00(1.00)$ & $6.22(0.44)$ \\
\hline UNA3: CML2 + Online IS $(\mathrm{n}=8)$ & $5.75(0.89)$ & $5.87(0.64)$ \\
\hline UNA4: CML2 + Ubiquitous IS $(\mathrm{n}=8)$ & $5.63(1.06)$ & $6.00(0.00)$ \\
\hline UNA5: Total CML1 $(\mathrm{n}=18)$ & $6.06(0.80)$ & $6.00(0.91)$ \\
UNA6: Total CML2 ( $\mathrm{n}=16)$ & $5.69(0.95)$ & $5.94(0.44)$ \\
UNA7: Total Online IS ( $\mathrm{n}=17)$ & $5.94(0.75)$ & $5.82(0.95)$ \\
UNA8: Total Ubiquitous IS $(\mathrm{n}=17)$ & $5.82(1.02)$ & $6.12(0.33)$ \\
\hline UNA9: Total $(\mathrm{n}=34)$ & $\mathbf{5 . 8 8}(\mathbf{0 . 8 8})$ & $\mathbf{5 . 9 7}(\mathbf{0 . 7 2})$ \\
\hline
\end{tabular}

Consistency: I think that this model of type [CML1/2] is consistent as such with regard to Situation [1/2].

\begin{tabular}{l|c|c} 
CON1: CML1 + Online IS ( $\mathrm{n}=9)$ & $5.78(0.67)$ & $5.67(1.00)$ \\
\hline CON2: CML1 + Ubiquitous IS (n=9) & $5.78(1.20)$ & $6.22(0.83)$ \\
\hline CON3: CML2 + Online IS ( $\mathrm{n}=8)$ & $5.38(1.41)$ & $5.50(1.93)$ \\
\hline CON4: CML2 + Ubiquitous IS $(\mathrm{n}=8)$ & $5.13(0.84)$ & $5.75(1.04)^{*}$ \\
\hline CON5: Total CML1 $(\mathrm{n}=18)$ & $5.78(0.94)$ & $5.94(0.94)$ \\
CON6: Total CML2 ( $\mathrm{n}=16)$ & $5.25(1.13)$ & $5.62(1.50)^{*}$ \\
CON7: Total Online IS ( $\mathrm{n}=17)$ & $5.59(1.06)$ & $5.59(1.46)$ \\
CON8: Total Ubiquitous IS ( $\mathrm{n}=17)$ & $5.47(1.07)$ & $6.00(0.94)^{*}$ \\
\hline CON9: Total $(\mathrm{n}=34)$ & $\mathbf{5 . 5 3 ( 1 . 0 5 )}$ & $\mathbf{5 . 7 9}(\mathbf{1 . 2 3})$ \\
\hline
\end{tabular}

Comprehensibility: I think this model of type [CML1/2] is easy to understand with regard to Situation [1/2].

\begin{tabular}{l|l|l} 
COM1: CML1 + Online IS $(\mathrm{n}=9)$ & $6.11(0.78)$ & $6.44(0.73)$ \\
\hline COM2: CML1 + Ubiquitous IS $(\mathrm{n}=9)$ & $6.11(1.67)$ & $6.67(0.50)$ \\
\hline COM3: CML2 + Online IS ( $\mathrm{n}=8)$ & $6.25(0.71)$ & $6.63(0.74)$ \\
\hline COM4: CML2 + Ubiquitous IS ( $\mathrm{n}=8)$ & $5.75(1.39)$ & $6.63(0.52)$ \\
\hline COM5: Total CML1 $(\mathrm{n}=18)$ & $6.11(0.96)$ & $6.56(0.62)^{*}$ \\
COM6: Total CML2 (n=16) & $6.00(1.01)$ & $6.63(0.62)^{*}$ \\
COM7: Total Online IS (n=17) & $6.18(0.73)$ & $6.53(0.72)^{*}$ \\
COM8: Total Ubiquitous IS $(\mathrm{n}=17)$ & $5.94(1.25)$ & $6.65(0.49)^{*}$ \\
\hline COM9: Total $(\mathrm{n}=34)$ & $\mathbf{6 . 0 6}(\mathbf{1 . 0 1})$ & $\mathbf{6 . 5 9}(\mathbf{0 . 6 1})^{* *}$ \\
\hline
\end{tabular}

Extrinsic power of CML for individual understanding (EPIU): I think that this model of type [CML1/2] made it easy for me to create an individual understanding of the conceptual model regarding Situation [1/2].

\begin{tabular}{l|l} 
EPIU1: CML1 + Online IS ( $\mathrm{n}=9)$ & $5.78(0.67)$ \\
\hline EPIU2: CML1 + Ubiquitous IS ( $\mathrm{n}=9)$ & $5.56(1.88)$ \\
\hline EPIU3: CML2 + Online IS ( $\mathrm{n}=8)$ & $5.87(0.64)$ \\
\hline EPIU4: CML2 + Ubiquitous IS ( $\mathrm{n}=8)$ & $4.88(2.03)$ \\
\hline
\end{tabular}


Table 2. (Continued)

\begin{tabular}{l|l}
\hline EPIU5: Total CML1 $(\mathrm{n}=18)$ & $5.67(1.37)$ \\
EPIU6: Total CML2 $(\mathrm{n}=16)$ & $5.38(1.54)$ \\
EPIU7: Total Online IS ( $\mathrm{n}=17)$ & $5.82(0.64)$ \\
EPIU8: Total Ubiquitous IS $(\mathrm{n}=17)$ & $5.24(1.92)$ \\
\hline EPIU9: Total $(\mathrm{n}=34)$ & $\mathbf{5 . 5 3 ( 1 . 4 4 )}$ \\
\hline
\end{tabular}

Extrinsic power of CML for a shared (team) understanding (EPSU): I think that, in general, the external conceptual model of type [CML1/2] supports a shared understanding in our team with respect to Situation [1/2].

\begin{tabular}{l|l} 
EPSU1: CML1 + Online IS ( $\mathrm{n}=9)$ & $5.78(0.97)$ \\
\hline EPSU2: CML1 + Ubiquitous IS ( $\mathrm{n}=9)$ & $6.44(0.53)$ \\
\hline EPSU3: CML2 + Online IS ( $\mathrm{n}=8)$ & $5.63(1.41)$ \\
\hline EPSU4: CML2 + Ubiquitous IS ( $\mathrm{n}=8)$ & $6.25(1.17)$ \\
\hline EPSU5: Total CML1 ( $\mathrm{n}=18)$ & $6.11(0.83)$ \\
EPSU6: Total CML2 ( $\mathrm{n}=16)$ & $5.94(1.29)$ \\
EPSU7: Total Online IS ( $\mathrm{n}=17)$ & $5.71(1.16)$ \\
EPSU8: Total Ubiquitous IS ( $\mathrm{n}=17)$ & $6.35(0.86)$ \\
\hline EPSU9: Total ( $\mathrm{n}=34)$ & $\mathbf{6 . 0 3 ( 1 . 0 6 )}$
\end{tabular}

No effects can be found for unambiguity. However the subjects were confident that all CMs for all CMLs represent target situations on a high level. Further research is required to assess whether this confidence is stable or whether it is related to a lack of modeling experience. Nonetheless reassurance after receiving an explanation might indicate stable confidence. The mean value (5.53) for the extrinsic power of CML for individual understanding (EPIU) shows that all models are perceived important for creating internal conceptual models. Only use case diagrams / sequence diagrams provide less support. However, it might indicate that internal CM extracts structural information on concepts and relationships from an originating external CM but loses direct links to the external $\mathrm{CM}$ itself. This complex relationship requires further research.

By conducting an analysis of variance, we could not find any significant differences with regard to the extrinsic power of a CML for individual or shared (team) understanding by varying the factors CML and the type of IS. Thus, type of IS and CML as used in the current study have not influenced the extrinsic power of a CML for individual understanding or for a shared (team) understanding. However, mean values show differences for individual and shared understanding (EPIU9 and EPSU9) that are significant at the .06 level by applying a paired-sample t-test. This could be explained by different metrics that are used for the internal CM and shared understanding between team members. For individual CM a test person seems to have higher requirements than for shared understandings.

The overall high mean values for all variables are surprising because all test persons were modeling novices. No significant differences were found for the CML with ontologies / activity diagrams compared to the CML with use cases / sequence diagrams. This is unexpected because ontologies can be considered rather new tools for conceptual modeling. In contrast, CM based on use cases diagrams / sequence 
diagrams were significantly improved by explanations with respect to consistency and comprehensibility. CM represented by ontologies / activity diagrams did not gain from explanations for consistency but for comprehensibility only. Furthermore, comprehensibility of both types of CM for both IS types are significantly improved by verbal explanations. In summary, it can be argued that advantages exist of some CML types for particular types of IS (e.g., CML1 - UIS). Differences between extrinsic power of CML combinations for individual and shared understanding indicate that CML play different roles on individual and group levels. A tentative conclusion is that it is not important which grammar is used as long as it provides basic diagrammatic elements. This complex relationship between models and conceptual models needs further in-depth studies.

\section{Summary}

This research has investigated how conceptual modeling languages enhanced with verbal explanations can affect shared understanding of conceptual models between members of non-expert design teams. An empirical study was carried out in which two types of information systems (online and ubiquitous) were tested to assess whether narratives and diagrammatic conceptual modeling languages could provide rich information so that individuals would perceive them as being unambiguous, consistent, and comprehensible.

This research is one response to the need for empirical research on topics related to conceptual modeling [3]. However, further research involving field studies are required to demonstrate the application of the results to real world requirements engineering work. This study also raises further research questions; for example, would similar effects be found for expert groups. Further research is also required to expand the study to include formal ontology representation tools.

\section{References}

1. Stachowiak, H.: Allgemeine Modelltheorie. Springer, Heidelberg (1973)

2. Chakraborty, S., Sarker, S., Sarker, S.: An Exploration into the Process of Requirements Elicitation: A Grounded Approach. J. Assoc. Inf. Syst. 11, 212-249 (2010)

3. Burton-Jones, A., Meso, P.: The Effects of Decomposition Quality and Multiple Forms of Information on Novices' Understanding of a Domain from a Conceptual Model. J. Assoc. Inf. Syst. 9, 748-802 (2008)

4. CIO Update, http://www.cioupdate.com/insights/article.php/ 3813646/Why-Software-Development-Projects-Fail-Part-IVRelease.htm

5. Vlaar, P., van Fenema, P., Tiwari, V.: Cocreating Understanding and Value in Distributed Work: How Members of Onsite and Offshore Vendor Teams Give, Make, Demand, and Break Sense. Mis Quart. 32, 227-255 (2008)

6. Lin, L., Geng, X., Whinston, A.: A Sender-Receiver Framework for Knowledge Transfer. Mis Quart. 29, 197-219 (2005) 
7. Urquhart, C.: Exploring Analyst-Client Communication: Using Grounded Theory Techniques to Investigate Interaction in Informal Requirements Gathering. In: Lee, A., Liebenau, J., DeGross, J. (eds.) Information Systems and Qualitative Research, pp. 149181. Chapman \& Hall, London (1997)

8. Wand, Y., Storey, V.C., Weber, R.: Analyzing the Meaning of a Relationship. ACM Trans. Database Systems 24, 494-528 (1999)

9. Fowler, M.: Analysis Patterns, Reusable Object Models. Addison-Wesley, Longman, Amsterdam (1997)

10. Scheer, A.-W.: ARIS. Modellierungsmethoden, Metamodelle, Anwendungen. Springer, Berlin (1998)

11. Booch, G., Rambaugh, J., et al.: The Unified Modeling Language User Guide. AddisionWesley, Redwood City (1999)

12. Chen, P.: The Entity-Relationship Model-Toward a Unified View of Data. ACM Transactions on Database Systems 1, 9-36 (1976)

13. Bera, P., Krasnoperova, A., Wand, Y.: Using Ontology Languages for Conceptual Modeling. Journal of Database Management 21, 1-28 (2010)

14. Wand, Y., Monarchi, D., Parsons, J., Woo, C.: Theoretical foundations for conceptual modelling in information systems development. Decis Support Syst. 15, 285-304 (1995)

15. Storey, V.C., Fracchia, F., Müller, H.: Cognitive design elements to support the construction of a mental model during software exploration. Journal of Systems and Software 44, 171-185 (1999)

16. Burton-Jones, A., Meso, P.: Conceptualizing Systems for Understanding: An Empirical Test of Decomposition Principles in Object-Oriented Analysis. Inform. Syst. Res. 17, 3860 (2006)

17. Davidson, E.J.: Technology Frames and Framing: A Socio-Cognitive Investigation of Requirements Determination. Mis Quart. 26, 329-358 (2002)

18. Fodor, J.A., Pylyshyn, Z.W.: Connectionism and Cognitive Architecture: A Critical Analysis. In: Pinker, S., Mehler, J. (eds.) Connections and Symbols, pp. 3-71. MIT Press, Cambridge (1988)

19. Gentner, D., Stevens, A.L.: Mental Models. Lawrence Erlbaum Associates, Hillsdale (1983)

20. Kosslyn, S.M.: Image and Mind. Harvard University Press, Cambridge (1980)

21. Shepard, R.N., Metzler, J.: Mental rotation of three-dimensional objects. Science 171, 701-703 (1971)

22. Kuechler, W., Vaishnavi, V.: On theory development in design science research: anatomy of a research project. Eur. J. Inform. Syst. 17, 489-504 (2008)

23. Cannon-Bowers, J.A., Salas, E., Converse, S.: Shared mental models in expert team decision making. In: Castellan, N.J. (ed.) Individual and Group Decision Making, pp. 221246. Lawrence Erlbaum Associates, Hillsdale (1993)

24. McGrath, J.E.: Groups: Interaction and Performance. Prentice-Hall, Englewood Cliffs (1984)

25. Habermas, J.: The Theory of Communicative Action, Reason and the Rationalization of Society, vol. 1. Heinemann, London (1984)

26. Wittenbaum, G.M.: Putting communication into the study of group memory. Human Communication Research 29, 612-623 (2003)

27. Rasker, P., Post, W., Schraagen, J.: Effects of two types of intra-team feedback on developing a shared mental model in Command \& Control teams. Ergonomics 43, 1167$1189(2000)$ 
28. Stout, R.J., Cannon-Bowers, J.A., Salas, E., Milanovich, D.: Planning, shared mental models, and coordinated performance: An empirical link is established. Human Factors 41, 61-71 (1999)

29. Lyytinen, K., Yoo, Y.: Issues and Challenges in Ubiquitous Computing. Communication of the ACM 45, 62-65 (2002)

30. Maass, W., Janzen, S.: Pattern-based approach for designing with diagrammatic and propositional conceptual models. In: Jain, H., Sinha, A.P., Vitharana, P. (eds.) DESRIST 2011. LNCS, vol. 6629, pp. 192-206. Springer, Heidelberg (2011)

31. Mathiassen, L., Tuunanen, T., Saarinen, T., Rossi, M.: A Contingency Model for Requirements Development. Journal of the Assoication for Information Systems 8, 569597 (2007)

32. Gruber, T.R.: A translation approach to portable ontologies. Knowledge Acquisition 5, 199-220 (1993)

33. Noy, N.F., McGuinness, D.L.: Ontology Development 101: A Guide to Creating Your First Ontology. Technical Report and Stanford Medical Informatics Technical Report SMI-2001-0880, Stanford Knowledge Systems Laboratory (2001)

34. Cockburn, A.: Writing effective use cases. Addison-Wesley, Upper Saddle River (2001)

35. Fettke, P.: How Conceptual Modeling Is Used. Communications of the Association for Information Systems 25, 571-592 (2009) 